\title{
Two-way wavelength conversion for visible and infrared signal pulses
}

\author{
M. Saito*, H. Kimura, S. Nakamura \\ Department of Electronics and Informatics, Ryukoku University, Seta, Otsu 520-2194, Japan \\ *Corresponding author: e-mail: msaito@ rins.ryukoku.ac.jp
}

\begin{abstract}
:
Two-way wavelength conversion was achieved for visible and infrared signal pulses by using a phosphorescent disk. When a visible $(530 \mathrm{~nm})$ or infrared $(980 \mathrm{~nm})$ laser beam was focused on the disk, absorbed photons created bright spots that emitted both visible and infrared phosphorescence (upconversion and downconversion). Although the phosphorescence lasted for $1 \mathrm{~ms}$, a pulse signal of $1 \mathrm{MHz}(1 \mu$ s period) was detected successfully by circulating the bright spots on the disk (a time-space conversion method). In comparison with downconversion, upconversion was easier to achieve for high-frequency signals, since the two-photon absorption process prevented overlapping of adjacent spots by restricting the phosphorescence spot size.
\end{abstract}

\section{Highlights:}

Two-way wavelength conversion was achieved for visible and infrared optical pulses.

A single all-optical device realized both upconversion and downconversion.

A time-space conversion method was used to create short pulses from phosphorescence.

Pulse signals of $1 \mathrm{MHz}$ were converted successfully by rotating a phosphorescent disk.

Keywords:

Upconversion; Phosphorescence; Time-space conversion; Two-way device; All-optical device 


\section{Introduction}

Rare earth elements play an important role in many optical devices, e.g., solid-state and fiber lasers [1], fiber amplifiers [2], color displays and white illuminators (lamps, LEDs) [3], afterglow phosphors [4], etc. Advances in optical technology are still accelerating the needs for these elements in spite of their rarity. The needs for rare earth elements usually come from the fact that their excited states have a long lifetime (sub-millisecond or longer) [5]; e.g., a population inversion has to be attained in lasers and amplifiers, and a long-lasting phosphorescence is required in displays and glow-in-the-dark illuminators. Thermal and photochemical durabilities are also their advantages, since reliable performance and maintenance-free operation are of particular importance in communication devices and illuminators. These characteristics contrast with organic dyes, which generally possess a short excitation lifetime (nanoseconds or shorter) and readily degrade by photobleaching or thermal dissolution [6].

A two-way wavelength conversion is a distinctive function that is attainable with rare earth doped phosphors but unattainable with other dyes. According to the law of energy conservation, decreasing a photon energy (downconversion) is readily achievable, but increasing a photon energy (upconversion) needs a complicated process. Upconversion is usually induced by combination of multiple coherent photons, and hence, requires ultra-short pulses or a phase-matching process, e.g., a quantum-mechanical two-photon absorption or a harmonic generation. The long lifetime of rare earth elements, however, realizes an upconversion process that requires no complicated optical systems [7]. That is, an electron is excited to an intermediate state by absorbing a photon, and stays at that state for a while (during a lifetime of that state) waiting for another photon arrival. Then the electron is excited to a higher energy level by absorbing a second photon and emits a high energy photon.

Since the early days of the upconversion research [8], a variety of phosphors have been 
developed by doping rare earth elements in crystals (powders) [9- 11], glass ceramics [12], glasses [13, 14], or glass fibers (waveguides) [15, 16]. Most phosphors emit green or red light, and some emit violet [17] or white (red-green-blue, RGB) light [18- 20]. Research interests are currently directed to enhancement of the phosphorescence efficiency or brightness, e.g., fabrication of nano-sized particles [21-23] or surface-plasmonic enhancement of phosphorescence [24, 25]. By using these upconversion phosphors, researchers have developed various optical devices [26- 29] including 3-D displays [30, 31]. In recent years, applications to biotechnology have been studied extensively [32- 35].

In this study, we used an upconversion phosphor to construct a two-way wavelength converter. Optical communication systems are currently operated in various spectral ranges, e.g., the $1300-1600 \mathrm{~nm}$ band for the long-haul transmission systems, the $800-1000 \mathrm{~nm}$ band for the wire-less networks, the red band for the polymer-based interconnections, and the entire visible band (white light) for the mobile communication systems based on LED illuminators. All-optical wavelength conversion is desired to interconnect these different communication systems. As a first step to achieving the two-way conversion, we conducted experiments by using $\mathrm{Yb}^{3+}$-Er ${ }^{3+}$ co-doped $\mathrm{Gd}_{2} \mathrm{O}_{2} \mathrm{~S}$ powder (Phosphor Technology, PTIR545/F). Figure 1 shows the emission processes of this phosphor [5]. $\mathrm{Er}^{3+}$ is responsible for emission, and $\mathrm{Yb}^{3+}$ facilitates the two-photon absorption because of its high infrared absorbance $[8,9]$. In the upconversion process, infrared photons in the $1000 \pm 80 \mathrm{~nm}$ range are absorbed, and phosphorescence occurs in the $540 \pm 10 \mathrm{~nm}$ range (green). In the downconversion process, green light excites electrons and induces infrared emission.

As mentioned earlier, a long lifetime of the excitation states is essential for achieving an efficient wavelength conversion. The long lifetime inevitably causes afterglow with a long decay time, which is preferred for uses in nonelectric illuminators [4, 36], imagers [37] and tracers [38]. As regards the wavelength conversion, however, the long lifetime is unfavorable 
since it restricts the signal frequency or bit-rate; i.e., the afterglow causes an overlap of successive pulses. When the pulse frequency of an excitation signal exceeds $1 \mathrm{kHz}(1 \mathrm{kbit} / \mathrm{s})$, for example, phosphorescence becomes continuous and carries no trace of signal pulses. In a previous paper [39], we proposed a time-space conversion method to realize a fast-response upconversion. In this method, optical pulses are irradiated on a disk containing the phosphor, as shown in Fig. 2(a), and create a bright spot array as the disk rotates. By picking up phosphorescence from these spots, signal pulses can be reproduced regardless of the afterglow time. A pulse signal upconversion from 940 to $530 \mathrm{~nm}$ was demonstrated successfully in the previous experiment. In the current experiment, we examine the validity of this method in the downconversion process to demonstrate the feasibility of the two-way conversion. We also examine whether a pulse signal of $980 \mathrm{~nm}$ can induce an efficient upconversion, since this wavelength is more suitable than $940 \mathrm{~nm}$ for matching the input and output signal wavelengths.

\section{Optical system}

Figure 2(a) shows the optical system that was constructed for the two-way wavelength conversion. The signal source was a laser diode of $980 \mathrm{~nm}$ (output power: $900 \mathrm{~mW}$ ) or a frequency-doubled Nd:YAG laser of $530 \mathrm{~nm}(300 \mathrm{~mW})$. The laser beam was modulated by using an acousto-optic modulator to generate a pulse signal of 1-3000 kHz. Figure 2(b) shows a $980 \mathrm{~nm}$ signal that was measured at the exit end of the input fiber (a silica glass fiber of 200 $\mu \mathrm{m}$ diameter). As the arrow shows, the detector signal varied between 40 and 400 (arbitrary unit), and hence, the modulation factor was $(400-40) /(400+40)=82 \%$ at this frequency. The average optical power was $260 \mathrm{~mW}$. Figure 2(c) shows the maximum and minimum of the signal intensity that were measured at various modulation frequencies. Although the average signal power was unchanged, the modulation factor decreased notably beyond $1 \mathrm{MHz}$ because 
of the operation limit of the acousto-optic modulator. This input beam was imaged on the phosphor disk (1:1 magnification) by using a lens. The upconversion phosphorescence of the disk was picked up with another couple of lens and optical fiber, which were placed symmetrically with the input optical system. When the downconversion experiment was conducted with the $530 \mathrm{~nm}$ light, the ends of the two fibers were changed with one another, as shown by dashed lines in Fig. 2(a). Consequently, the signal beam progressed reversely in the two lens systems (two-way transmission). The modulated $530 \mathrm{~nm}$ light exhibited a similar signal shape and a similar frequency characteristic as those shown in Figs. 2(b) and 2(c). The average signal power was $50 \mathrm{~mW}$.

The phosphor disk consisted of two polycarbonate disks (120 mm diameter) that sandwiched the phosphor powder [39]. As Fig. 2(a) shows, the input light created a series of phosphorescent spots on the disk that rotated at 40 revolution/s. In a previous experiment, we observed a delay signal by picking up the phosphorescence at a downstream position of the spot flow [39]. In the current experiment, however, the output signal (phosphorescence) was picked up at the irradiation point of the input signal, since the input and output optical systems had to be equivalent in the two-way transmission device.

A gray line in Fig. 3(a) shows an upconversion signal spectrum that was measured at the exit end of the output fiber. A pronounced peak at $980 \mathrm{~nm}$ was attributed to the input light that was scattered at the disk surface. Broad phosphorescence peaks are visible in the wavelength ranges of $530-550,650-680,800-860$, and $920-1080 \mathrm{~nm}$, which correspond to electronic transitions between several different energy levels [5]. To extract a green signal from this phosphorescence, a color filter was inserted in front of the output fiber, as shown in Fig. 2(a). As the black line in Fig. 3(a) shows, this filter was opaque in the $600-1100 \mathrm{~nm}$ wavelength range, and hence, removed the unwanted noise light, i.e., the scattered input light and the phosphorescence at the other wavelengths. 
In the downconversion experiment, the input and output fibers were connected to the opposite side of the converter, as shown by dashed lines in Fig. 2(a). A modulated green laser beam was delivered by the input fiber and irradiated the phosphor disk from the lower side. The color filter did not hinder this signal transmission, since it was transparent in the green spectral range. The gray line in Fig. 3(b) shows a phosphorescence spectrum that was measured through the output fiber on the other side of the converter. The spectrum was similar to that in Fig. 3(a) except that a peak of the scattered input light appeared at $530 \mathrm{~nm}$. To eliminate unwanted spectral lines, another color filter was inserted in the optical path (the upper side of the converter). As the black line in Fig. 3(b) shows, this filter possessed a band-pass transmission characteristic for the $900-1020 \mathrm{~nm}$ wavelength range. Insertion of this filter caused no problem to the other input signal $(980 \mathrm{~nm})$, since it was transmissive at the signal wavelength.

Figures 3(c) and 3(d) show the input and output signal spectra in the upconversion and downconversion processes. As mentioned earlier, the signal upconversion capability was demonstrated by using a $940 \mathrm{~nm}$ laser diode in the previous experiment [39]. In the downconversion process, however, phosphorescence was stronger at $980 \mathrm{~nm}$ than $940 \mathrm{~nm}$, as shown in Fig. 3(b). A $980 \mathrm{~nm}$ laser was therefore used as an input signal source in the current experiment. As Figs. 3(c) and 3(d) show, the input and output signal wavelengths were reversed successfully in the current two-way conversion process.

\section{Experiment and discussion}

Figures 4(a)-4(c) show the upconversion signals that were measured by a photodetector at the output fiber end. These signals oscillated up and down across $0 \mathrm{~V}$, since the detector output was AC-coupled for signal amplification. When the modulation frequency of the input infrared signal was 20 or $50 \mathrm{kHz}$, a clear output signal was detected. The output signal intensity 
decreased gradually as the frequency increased, and the pulse signal became undetectable beyond $200 \mathrm{kHz}$. Note that the input signal had a sufficient modulation factor (>80\%) below 1 MHz, as shown in Fig. 2(c). The signal pulses were therefore assumed to have disappeared in the wavelength conversion process.

Let us consider what determined the frequency limit of the wavelength conversion. In the current experiment, the rotation rate of the phosphor disk (120 mm diameter) is 40 revolution/s, and the bright spots draw a circular trajectory of $110 \mathrm{~mm}$ diameter. The linear velocity of the spots is therefore $14 \mathrm{~m} / \mathrm{s}$. If the input signal frequency is $f$, the distance between two adjacent spots is $14 / f$. At $200 \mathrm{kHz}$ (the detection limit), for example, the distance between spot centers is evaluated to be $70 \mu \mathrm{m}$. The bright spot diameter is therefore assumed to be smaller than $\sim 70 \mu \mathrm{m}$, since otherwise bright spots overlap one another creating a continuous phosphorescence signal (no modulation). This assumed spot size is smaller than the image size of the fiber end (200 $\mu \mathrm{m}$ diameter) or the input beam diameter. Probably the upconversion phosphorescence is emitted only at the beam center, since the two-photon absorption is difficult to take place at the outer region where the photon density (the input light intensity) is low.

Next, the input signal of $530 \mathrm{~nm}$ was transmitted in the reverse direction to generate a downconversion signal. Figure 4(d) shows the output signal that was detected at $20 \mathrm{kHz}$. As Fig. 4(e) shows, however, the signal became undetectable at $50 \mathrm{kHz}$. When the detector signal was measured by DC-coupling, a flat signal with no modulation appeared, as shown in Fig. 4(f). This result indicated that the pulse signal vanished not because the phosphorescence (the output signal) was weak but because the bright spots overlapped one another.

Although the $200 \mathrm{kHz}$ signal is detectable in the upconversion process, the modulation frequency is limited below $50 \mathrm{kHz}$ in the downconversion process. This fact is related to the excitation mechanism of the phosphor. As mentioned earlier, the upconversion 
phosphorescence is restricted to a narrow region in which two-photon absorption takes place. By contrast, the downconversion phosphorescence takes place in the entire region to which the input beam is collected. In addition, the powdery phosphor heavily scatters the input light and expands the input beam diameter $(200 \mu \mathrm{m})$. The spot distance is evaluated to be $280 \mu \mathrm{m}$, when the modulation frequency is $50 \mathrm{kHz}$ and the rotation rate is 40 revolution/s. The diameter of the bright spots probably exceeds this spot distance in the downconversion process.

The consideration above suggested that the modulation frequency could be raised by either increasing the disk rotation rate or decreasing the input beam diameter. When the rotation rate was raised to 80 revolution/s, however, no stable signal was attainable, since the disk vibrated heavily as the rotation rate increased. To reduce the input beam diameter, we removed the optical fibers and irradiated the modulated laser beam directly to the phosphor disk. The focused beam diameter was $20-30 \mu \mathrm{m}$ on the disk surface.

Figure 5(a) shows an upconversion signal that was detected by irradiating a $980 \mathrm{~nm}$ signal directly to the phosphor disk. A clear sinusoidal signal was visible at $1 \mathrm{MHz}$. The bright spot distance was evaluated to be $14 \mu \mathrm{m}$ at this modulation frequency, since the disk rotated at 40 revolution/s. It was assumed that only the central portion of the input beam induced the two-photon absorption and the green phosphorescence.

Figure 5(b) shows a downconversion signal that was measured by focusing a $530 \mathrm{~nm}$ beam on the phosphor disk. Although a signal of $1 \mathrm{MHz}$ was detectable, its shape was heavily distorted. This result also indicated that a superior performance was attainable in the upconversion process than the downconversion process.

A reason for the spot expansion is the scattering by the phosphor powder (average size: 3 $\mu \mathrm{m})$. If a transparent upconversion glass is used [40], the modulation frequency can be raised in the downconversion process. The increase of the disk rotation rate is also effective to improve the frequency characteristic. The current disk that was made by adhering two DVD 
disks vibrated heavily during the high-speed rotation process because of a poor mechanical balance. Recent technology has developed polygonal mirrors that are driven by an electric motor with a rotation rate exceeding 600 revolution/s [41]. We are currently trying to use this technique in our phosphor disk. Also, this polygonal mirror technique is usable in another way; i.e., the time-space conversion is achievable by scanning a signal beam on a fixed phosphor plate instead of rotating the phosphor disk. This configuration seems more suitable for creating a compact optical device.

In solid state lasers and fiber amplifiers, short pulses are generated or amplified by using rare earth elements. The long lifetime does not matter in these devices, since stimulated emission generates a strong pulse that overcomes the afterglow effect. If stimulated emission is induced by placing a phosphor in a resonator, a fast wavelength conversion may be achievable without the use of rotation mechanism.

\section{Conclusion}

Two-way wavelength conversion was conducted by using a rare earth doped phosphor. To eliminate the afterglow effect that prevented the fast response, a pulse signal in the time domain was converted to a phosphorescent spot array in the space domain. Signal pulses of 530 and $980 \mathrm{~nm}$ wavelengths were converted to one another at $1 \mathrm{MHz}$ modulation frequency. The time-space conversion method will be useful to interconnect optical networks that are operated at various wavelengths.

\section{Acknowledgements}

This research was supported by a research grant (A-STEP) from Japan Science and Technology Agency. A part of this research was supported by Grant-in-Aid for Scientific Research from Japan Society for the Promotion of Science. 


\section{References}

[1] C.J. Koester, E. Snitzer, Appl. Opt. 3 (1964) 1182.

[2] M. Nakazawa, Y. Kimura, K. Suzuki, Appl. Phys. Lett. 54 (1989) 295.

[3] Y. Narukawa, Opt. Photon. News, 15 (April 2004) 24.

[4] T. Matsuzawa, Y. Aoki, N. Takeuchi, Y. Murayama, J. Electrochem. Soc. 143 (1996) 2670.

[5] S. Shionoya, W.M. Yen (Eds.), Phosphor Handbook, CRC Press, Boca Raton, 1999, Chapter 3.

[6] M. Saito, T. Nishimura, K. Sakiyama, S. Inagaki, AIP Adv. 2 (2012) 042118.

[7] F. Auzel, Chem. Rev. 104 (2004) 139.

[8] L.F. Johnson, J.E. Geusic, H.J. Guggenheim, T. Kushida, S. Shingh, L.G. Uitert, Appl. Phys. Lett. 15 (1969) 48.

[9] T. Kano, H. Yamamoto, Y. Otomo, J. Electrochem. Soc. 119 (1972) 1561.

[10] H.X. Zhang, C.H. Kam, Y. Zhou, X.Q. Han, S. Buddhudu, Y.L. Lam, Opt. Mater. 15 (2000) 47.

[11] N. Rakov, R.B. Guimarães, G.S. Maciel, Appl. Phys. B, 98 (2010) 435.

[12] Y. Kishi, S. Tanabe, S. Tochino, G. Pezzotti, J. Am. Ceram. Soc. 88 (2005) 3423.

[13] D.C. Yeh, W.A. Sibley, M. Suscavage, M.G. Drexhage, J. Appl. Phys. 62 (1987) 266.

[14] S. Tanabe, K. Hirao, N. Soga, J. Non-Cryst. Solids, 122 (1990) 79.

[15] D.C. Hanna, R.M. Percival, I.R. Perry, R.G. Smart, J.E. Townsend, A.C. Tropper, Opt. Commun. 78 (1990) 187.

[16] J.L. Jackel, A. Yi-Yan, E.M. Vogel, A.V. Lehmen, J.J. Johnson, E. Snitzer, Appl. Opt. 31 (1992) 3390.

[17] T.Y. Fan and R.L. Byer, J. Opt. Soc. Am. B, 3 (1986) 1519.

[18] J.P. Ziel, L.G. Uitert, W.H. Grodkiewicz, R.M. Mikulyak, J. Appl. Phys. 60 (1986) 4262. 
[19] Y. Mita, K. Hirama, N. Ando, H. Yamamoto, S. Shionoya, J. Appl. Phys. 74 (1993) 4703.

[20] J.H. Chung, S.Y. Lee, K.B. Shim, J.H. Ryu, Appl. Phys. Express, 5 (2012) 052602.

[21] A. Patra, C.S. Friend, R. Kapoor, P.N. Prasad, J. Phys. Chem. B, 106 (2002) 1909.

[22] D. Matsuura, Appl. Phys. Lett. 81 (2002) 4526.

[23] Y. Li, T. Liu, Y. Du, Appl. Phys. Express, 5 (2012) 08650.

[24] T. Aisaka, M. Fujii, S. Hayashi, Appl. Phys. Lett. 92 (2008) 132105.

[25] W. Feng, L.-D. Sun, C.-H. Yan, Chem. Commun. (2009) 4393.

[26] M.P.L. Flohic, J.Y. Allain, G.M. Stéphan, G. Mazé, Opt. Lett. 19 (1994) 1982.

[27] R. Scheps, Prog. Quant. Electr. 20 (1996) 271.

[28] M. Saito, Y. Takahashi, Opt. Lett. 33 (2008) 1687.

[29] T. Lu, L. Yang, R.V.A. Loon, A. Polman, K.J. Vahala, Opt. Lett. 34 (2009) 482.

[30] J.D. Lewis, C.M. Verber, R.B. Mcghee, IEEE Trans. Electron. Devices, 18 (1971) 724.

[31] E. Downing, L. Hesselink, J. Ralston, R. Macfarlane, Science, 273 (1996) 1185.

[32] F. Rijke, H. Zijlmans, S. Li, T. Vail, A.K. Raap, R.S. Niedbala, H.J. Tanke, Nat. Biotechnol. 19 (2001) 273.

[33] P.L.A.M. Corstjens, S. Li, M. Zuiderwijk, K. Kardos, W.R. Abrams, R.S. Niedbala, H.J. Tanke, IEE Proc. Nanobiotechnol. 152 (2005) 64.

[34] K. Kuningas, H. Päkkilä, T. Ukonaho, T. Rantanen, T. Lövgren, T. Soukka, Clin. Chem. 53 (2007) 145.

[35] P. Zhang, W. Steelant, M. Kumar, M. Scholfield, J. Am. Chem. Soc. 129 (2007) 4526.

[36] M. Saito, N. Adachi, H. Kondo, Opt. Express, 15 (2007) 1621.

[37] S.F. Lim, R. Riehn, W.S. Ryu, N. Khanarian, C.-K. Tung, D. Tank, R.H. Austin, Nano Lett. 6 (2006) 169.

[38] M. Kamimura, D. Miyamoto, Y. Saito, K. Soga, Y. Nagasaki, Langmuir, 24 (2008) 8864.

[39] M. Saito, S. Nakamura, T. Kita, Appl. Phys. Lett. 99 (2011) 191101. 
[40] Sumita Optical Glass Inc. (Japan), YAGLASS,

http://www.sumita-opt.co.jp/ja/products/optical/yaglass.html.

[41] Lincoln Laser Corp. (USA), Polygonal Mirrors, http://www.lincolnlaser.com/products/mirrors.cfm. 


\section{Figure captions}

Fig. 1. Energy levels of an $\mathrm{Yb}^{3+}-\mathrm{Er}^{3+}$ co-doped phosphor. Numerals beside the arrows denote the absorption or emission wavelengths that correspond to the electronic transition.

Fig. 2. (a) Optical system for conducting the two-way wavelength conversion of signal pulses. (b) Input signal shape that was measured at the input fiber end. The original continuous-wave (CW) laser beam was modulated at $10 \mathrm{kHz}$ by using an acousto-optic modulator. (c) Modulation efficiency of the input signal. The open and closed circles show the maximum and minimum values of the input signal. The arrow corresponds to that in (b).

Fig. 3. (a) Upconversion or (b) downconversion phosphorescence spectrum (gray line) that was measured at the output fiber end with no color filter. The black line shows the transmission spectrum of the color filter that was placed in front of the output fiber. A pronounced peak at (a) 980 or (b) $530 \mathrm{~nm}$ wavelength originated from the scattered input signal (excitation light). (c, d) Signal light spectra that were measured at the input and output fiber ends. The output light spectrum was measured through the color filter shown in (a) or (b).

Fig. 4. Output signals in the $(\mathrm{a}-\mathrm{c})$ upconversion and $(\mathrm{d}-\mathrm{f})$ downconversion processes. Input signal frequencies are shown in the figures. The detector signal was AC-coupled to the oscilloscope except for the background noise in (f) that was monitored by DC-coupling.

Fig. 5. Output signals that were measured by removing both the input and output fibers. The input signal frequency was $1 \mathrm{MHz}$. (a) shows an upconversion signal that was detected through the color filter shown in Fig. 3(a). (b) shows a downconversion signal that was detected through the color filter shown in Fig. 3(b). 


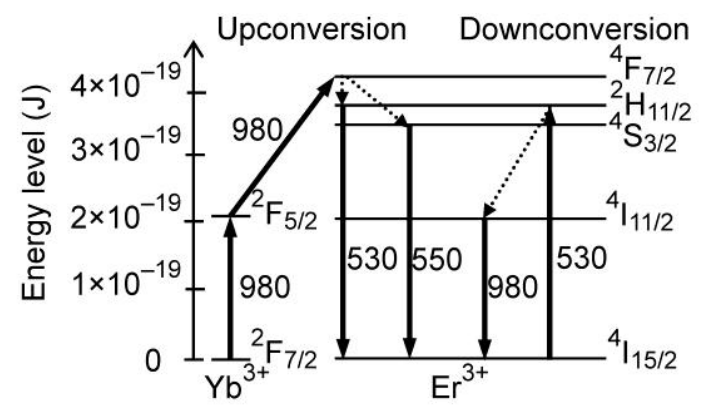

Fig. 1. Energy levels of an $\mathrm{Yb}^{3+}-\mathrm{Er}^{3+}$ co-doped phosphor. Numerals beside the arrows denote the absorption or emission wavelengths that correspond to the electronic transition. 


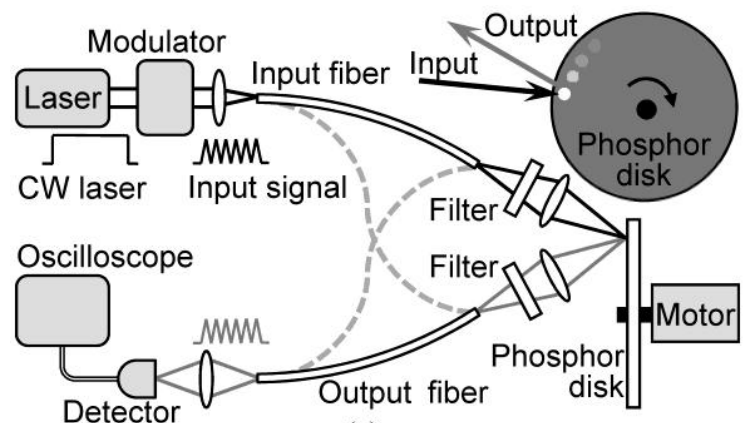

(a)

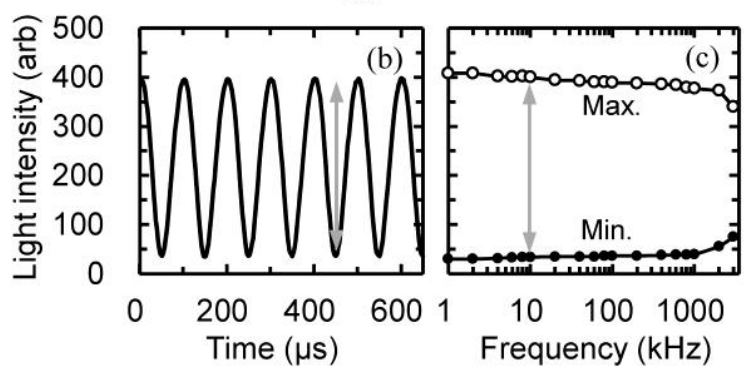

Fig. 2. (a) Optical system for conducting the two-way wavelength conversion of signal pulses. (b) Input signal shape that was measured at the input fiber end. The original continuous-wave $(\mathrm{CW})$ laser beam was modulated at $10 \mathrm{kHz}$ by using an acousto-optic modulator. (c) Modulation efficiency of the input signal. The open and closed circles show the maximum and minimum values of the input signal. The arrow corresponds to that in (b). 

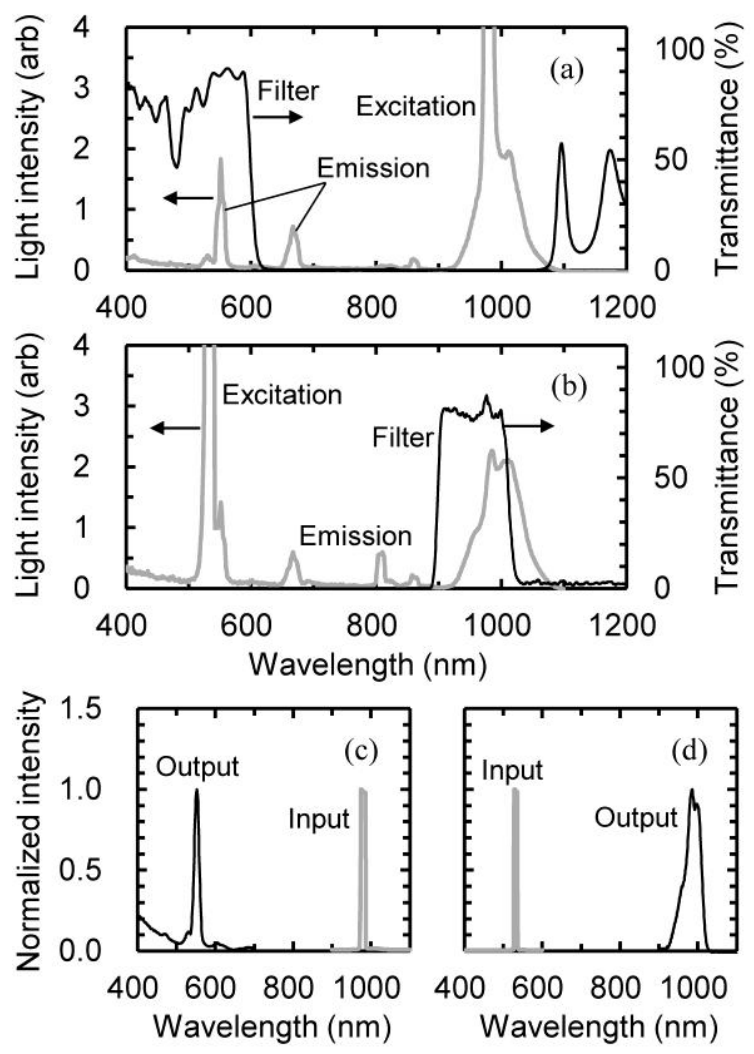

Fig. 3. (a) Upconversion or (b) downconversion phosphorescence spectrum (gray line) that was measured at the output fiber end with no color filter. The black line shows the transmission spectrum of the color filter that was placed in front of the output fiber. A pronounced peak at (a) 980 or (b) $530 \mathrm{~nm}$ wavelength originated from the scattered input signal (excitation light). (c, d) Signal light spectra that were measured at the input and output fiber ends. The output light spectrum was measured through the color filter shown in (a) or (b). 

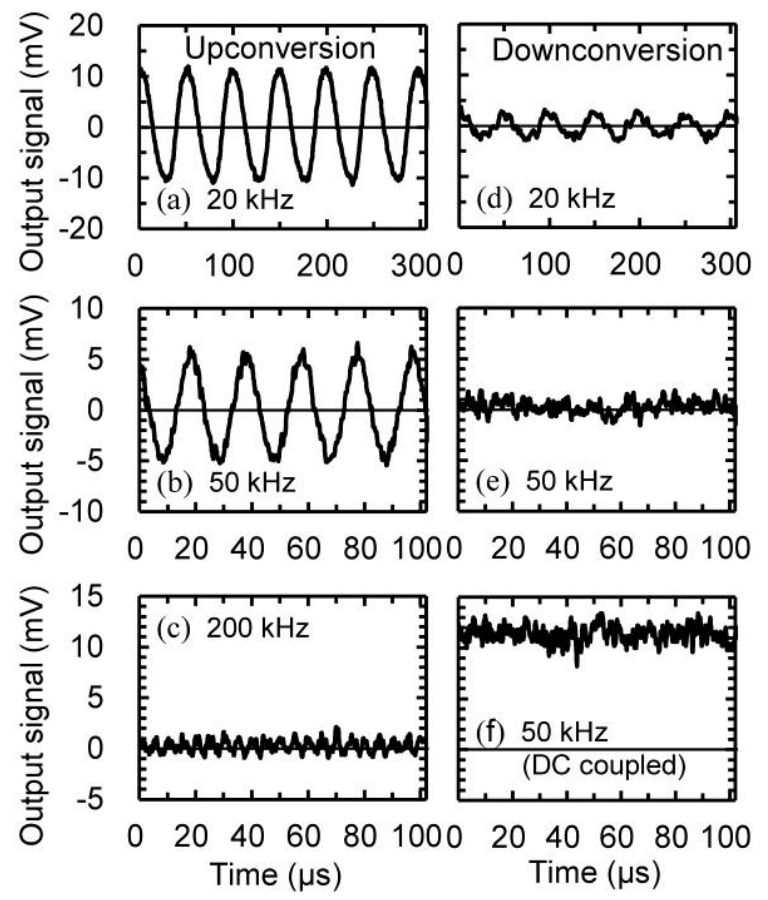

Fig. 4. Output signals in the $(a-c)$ upconversion and $(d-f)$ downconversion processes. Input signal frequencies are shown in the figures. The detector signal was AC-coupled to the oscilloscope except for the background noise in (f) that was monitored by DC-coupling. 


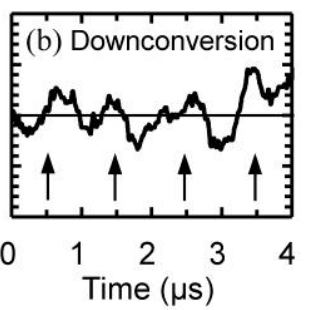

Fig. 5. Output signals that were measured by removing both the input and output fibers. The input signal frequency was $1 \mathrm{MHz}$. (a) shows an upconversion signal that was detected through the color filter shown in Fig. 3(a). (b) shows a downconversion signal that was detected through the color filter shown in Fig. 3(b). 